\title{
AQUATIC INTERFACES: A HYDRODYNAMIC AND ECOLOGICAL PERSPECTIVE
}

ANDREA MARION, Associate Professor, Department of Industrial Engineering, University of Padua, Padua, Italy

Email: andrea.marion@unipd.it (author for correspondence)

VLADIMIR NIKORA (IAHR Member), Professor, School of Engineering, University of Aberdeen, Aberdeen, United Kingdom

Email:v.nikora@abdn.ac.uk

SARA PUIJALON, CNRS Associate Scientist, UMR 5023 Laboratory of Ecology of Natural and Man-impacted Hydrosystems, CNRS, Université Lyon 1, ENTPE, Lyon, France

Email: sara.puijalon@univ-lyon1.fr

TJEERD BOUMA, Senior Scientist, Department of Spatial Ecology, Royal Netherlands Institute for Sea Research, Yerseke, The Netherlands

Email: tjeerd.bouma@nioz.nl

KATINKA KOLL (IAHR Member), Senior Lecturer, Leichtweiß-Institute for Hydraulic Engineering and Water Resources, TU Braunschweig, Braunschweig, Germany

Email:katinka.koll@tu-bs.de

FRANCESCO BALLIO (IAHR Member), Professor, Department of Civil and Environmental Engineering, Politecnico di Milano, Milano, Italy

Email: francesco.ballio@polimi.it

SIMON TAIT (IAHR Member), Professor, School of Engineering, Design and Technology, University of Bradford, Bradford, United Kingdom, or affiliation as appropriate

Email: s.tait@bradford.ac.uk

MATTIA ZARAMELLA, Technical Director, WET Water and Environmental Engineering Srl, Castelfranco Veneto, Italy

Email: zaramella@wetengineering.com 
ALEXANDER SUKHODOLOV, Senior Scientist, Department 1 Ecohydrology, Leibniz Institute for Freshwater Ecology and Inland Fisheries, Berlin, Germany

Email:alex@igb-berlin.de

MATTHEW O'HARE, Senior Scientist, Centre for Ecology \& Hydrology, Edinburgh, United Kingdom

Email:moha@ceh.ac.uk

GERALEDENE WHARTON, Professor, School of Geography, Queen Mary University of London, London, United Kingdom

Email: g.wharton@qmul.ac.uk

JOCHEN ABERLE, Professor, Department of Hydraulic and Environmental Engineering, Norwegian University of Science and Technology, Trondheim, Norway

Email: jochen.aberle@ntnu.no

MATTEO TREGNAGHI, Project Manager, Department of Industrial Engineering, University of Padua, Padua, Italy

Email: matteo.tregnaghi@unipd.it

PETER DAVIES, Professor, School of Engineering, Physics \& Mathematics, University of Dundee, Dundee, United Kingdom, or affiliation as appropriate

Email:p.a.davies@dundee.ac.uk

HEIDI NEPF, Professor, Department of Civil and Environmental Engineering, Massachusetts Institute of Technology, Cambridge, Massachusetts

Email: hmnepf@mit.edu

GARY PARKER, Professor, Department of Civil Engineering, University of Illinois, Urbana, Illinois

Email: parkerg@illinois.edu

BERNHARD STATZNER, Research Director - retired, UMR 5023 Laboratory of Ecology of Natural and Man-impacted Hydrosystems, CNRS, Université Lyon 1, ENTPE, Lyon, France

Email: bernhard.statzner@orange.fr

Running Head: Biophysical interfaces 


\title{
AQUATIC INTERFACES: A HYDRODYNAMIC AND ECOLOGICAL PERSPECTIVE
}

\begin{abstract}
Ecologically-appropriate management of natural and constructed surface water bodies has become increasingly important given the growing anthropogenic pressures, statutory regulations, and climatechange impacts on environmental quality. The development of management strategies requires that a number of knowledge gaps be addressed through interdisciplinary research efforts particularly focussing on the water-biota and water-sediment interfaces where most critical biophysical processes occur. This forum paper discusses the current state of affairs in this field and highlights potential paths to resolve critical issues, such as hydrodynamically-driven mass transport processes at interfaces and associated responses of organisms through the development of traits. The roles of experimental methods, theoretical modelling, statistical tools, and conceptual upscaling methods in future research are discussed from both engineering and ecological perspectives. The aim is to attract the attention of experienced and emerging hydraulic and environmental researchers to this research area, which is likely to bring new and exciting discoveries at the discipline borders.
\end{abstract}

Keywords: Flow-biota interactions, hydrodynamics, interfaces, sediments, wetlands

Running Head: Biophysical interfaces

\section{Introduction}

The changes to national and international statutory policies in response to emerging priorities of sustainable use of water resources and challenges of climate change require advanced understanding of ecological issues and its practical implementation in environmental management strategies to protect natural aquatic environments (e.g., US Clean Water Act 1972 and EU Water Framework Directive - WFD 2000 and subsequent amendments, ICCP rep. 2007, EU Floods Directive 2007, Global Water Security Declaration 2013). This target can only be achieved with in-depth knowledge of the interrelations between physical and biological processes, i.e., it is inevitable to address existing knowledge gaps through interdisciplinary research covering the fields of fluid mechanics, ecology, geomorphology, hydrology and biomechanics (e.g., Rodriguez-Iturbe 2000, Nikora 2010, Nepf 2012) taking also into account societal, cultural and economic aspects (e.g., Freude and Fluss 2007). However, interdisciplinary research is often avoided by researchers due to the traditional thematic structure of education and research, intrinsic difficulties in developing common 'cultural' ground and terminology, and complications in obtaining research funding for interdisciplinary research. Hence, the integration of discipline-related perspectives into a 
unifying multidisciplinary research platform or framework constitutes a key contemporary challenge in order to provide solutions for some of the most intriguing questions that humanity is facing in its relationship with the natural hydro-bio-sphere.

In this forum paper, we address this topic by highlighting various issues related to interdisciplinary research in the field of aquatic ecosystems. The recognition and implementation of hydrodynamics in biological studies has hastened the development of theoretical and applied issues in aquatic ecology (e.g., Statzner et al. 1988). At the same time it is important to provide hydraulic researchers and engineers with knowledge of ecological principles so that they can be adequately considered in the development of engineering solutions. This is particularly timely as hydraulic engineers play a crucial role in the development of sustainable management strategies of natural and constructed surface water bodies. A key to succeed in this challenging task is to develop in-depth understanding of interactions between water, biota, and sediments. Most of the critical biophysical processes occur at the water-biota and water-sediments interfaces thus calling for fundamental insights into the interrelations of physical and biological processes at a scale of the smallest biophysical units and the upscaling of this knowledge to larger scales. However, the creation of the unifying platform so urgently needed by the research community is delayed by the slower advances in the understanding of flow-biota interactions compared to other topics such as flow-sediment interactions. Among factors limiting the progress in this area are difficulties in measuring physical, biological, and biochemical quantities at the spatial and temporal scales of the organisms and sediment grains, including biomechanical properties that evolved in specific ecological conditions and their effects on the local interface morphology.

Many of these issues have been discussed extensively within a group of scientists and environmental practitioners and these discussions have led to the development of the EUInitial Training Network (PEOPLE - Marie Curie Action) - Hydrodynamic Transport in Ecologically Critical Heterogeneous Interfaces (HYTECH). The goal of this forum paper is to enlarge the audience of this discussion and hence to attract the attention of diverse research communities, in general, and the hydraulic engineering community in particular, to this important multidisciplinary issue.

The paper consists of eight sections. Section 2 outlines a set of fundamental concepts and keywords defining research topics in eco-hydraulics relevant to aquatic interfaces. Section 3 discusses the key outcomes of environmental fluid mechanics in explaining the hydrodynamics at fluid/sediment and fluid/biota interfaces. Section 4 offers an overview of the ecological perspective, highlighting the importance of interfacial processes for ecosystem formation and preservation. In Section 5, the issues of fluid-sediment interfaces are discussed, focussing on both processes occurring at and near the bed and the hyporheic processes acting within the river bed. Hydrodynamic and biological processes at water-biota interfaces are 
examined in Section 6. In Section 7, the case of wetlands, where nearly all of the most significant interfacial processes take place, is discussed, including a social perspective. Finally, the Conclusion section summarises the urgent needs and foreseeable targets of research on aquatic interfaces.

\section{Conceptual issues of eco-hydraulics relevant to biophysical interfaces}

Aquatic interfaces include a wide spectrum of boundaries of the water domains, characterized by spatial scales spanning several orders of magnitude (Gualtieri et al. 2013). In this discussion we focus on interfaces that are within the scales of river morphology, e.g., meander or wetland characteristic sizes, thus excluding larger interfaces such as the oceanic water-air interface or the water-sediment interface at catchment scales. The range of interfaces treated here are depicted and summarized, along with their typical spatial and temporal scales, in Fig. 1. Within this frame, interfaces can be defined as fairly narrow regions between two or more substances (phases) or between flow layers with distinctly different dynamic or physical properties (e.g., turbulent, transitional turbulent, and/or viscous flow domains, Stone 2010). Most relevant examples include water-sediment or water-biota or water-sediment-biota interfaces. An inherent property of interfaces in natural water bodies is that they are sites of steep gradients or maximum values of important physical and biochemical quantities (e.g., fluid velocity, turbulence intensity, viscous or turbulent stresses, concentration of substances, temperature, and light). The gradients of flow velocity and substance concentration at water-sediment and water-biota interfaces control diffusive momentum and mass fluxes and, thus, may determine the overall metabolic rates of individual organisms, their communities, and even the whole ecosystem units.

Understanding the transport mechanisms for inorganic and organic matter, including gases, solutes, colloids and solids in river flows and across bio-physical interfaces at different spatial and temporal scales is an essential step towards assessing the vulnerability of the natural environment to anthropogenic stresses. Typical stream interfaces at a small (micro-) scale are associated with inorganic, microbial, vegetation and/or animal structures such as sediment grains and clusters, biofilms, periphyton patches, and smaller invertebrates. At an intermediate (meso-) scale, comparable to the water depth, interfacial heterogeneities include sediment bedforms (e.g., ripples and dunes), patches of submerged and emergent plants, mussel aggregations, salmon redds, and upper layers of hyporheic zones. At larger (macro-) scales, comparable to the stream width, heterogeneities include large bedforms (e.g., alternate bars), large woody debris, vegetation patch mosaics, and deep layers of hyporheic zones. As the scale of consideration increases from micro- to meso- to macro- scale the research focus progressively moves from consideration of individual (mono-discipline) processes observed 
in well-defined experimental conditions towards large-scale set-ups and their models where multi-scale bio-physical interactions at the interfaces must be accounted for with appropriate theoretical upscaling frameworks, statistical tools and probabilistic methods.

The multi-scale nature of flow-biota interactions (Figure 1) requires advanced understanding of how the upscaling of fundamental processes from the elementary scale of organisms and grains to the larger scales can be achieved and represented quantitatively for modelling large-scale systems (e.g., at a river reach scale). The crucial role of heterogeneities in stream geometry and composition of mobile and immobile interfacial substances can be explored using a variety of innovative experimental and mathematical tools. The double (in time and space) averaging of fundamental momentum, mass transport, and energy equations allows researchers to formally extend the concepts of uniformity, steadiness and equilibrium, conventionally used in the time-(ensemble)-averaging framework, to locally-heterogeneous conditions (e.g., Finnigan 2000, Nikora 2010). The notions of uniformity, steadiness and equilibrium are useful concepts, which can lead to important simplifications of the mathematical approaches in complex cases by decoupling the mathematical terms associated with physical processes acting at well-separated scales. This permits, for instance, the application of simplified flow models when dealing with long-term morphological or ecological transitions, if the time scale separation of the relevant processes is significant. Simplifications can also be pursued when spatial upscaling is sought. Fundamental equations can, in principle, be averaged spatially (e.g., by employing a range of averaging operators and their parameters) which may help in accounting for multi-scale heterogeneity. Both temporal and spatial integrations lead to large-scale formulations at the expense of introduction of extra terms in the equations (due to non-linearity) for which appropriate closures are needed. Finding appropriate closures for these integrative terms is a great challenge of modern ecohydraulic research. The closures can, in principle, be identified rationally, by theoretical or phenomenological models, and experimentally, through direct experimental observations of individual processes. These two approaches can be supplemented with high-resolution numerical simulations.

Among the fundamental concepts underpinning eco-hydraulics, flow regime and temporal variability is very important. Hydraulics and hydrodynamics of surface water bodies have been of interest to humans since the early civilisations, due to the importance of water resources for human life combined with the need to find protection from the threats posed by extreme climatic events such as flood and debris flows. Extreme flows occurring with a return period of several decades usually activate intense sediment movement and, occasionally, create unsustainable conditions for river ecosystems, leading to the destruction of both interfacial habitats and existing ecological states. Extreme flood events often trigger major transitions towards a new system state and, therefore, their occurrences can be seen as 
determining natural ecosystem life spans. Between two destructive events, moderate and low flows determine the imposed conditions for the dynamics of populations, food webs, and species interactions, with the most important biochemical and biophysical processes occurring at the water-sediment-biota interfaces rather than within the main water domains. One of the fundamental issues of modern eco-hydraulics is, therefore, the fluid dynamics and mass transport associated with these interfaces.

Another critical issue to account for is biomechanical and morphological properties of biota living at water-sediment interfaces which reflect an evolutionary history that has imposed compromising solutions to multiple physical constraints. At slow flows (low Reynolds numbers), the organism morphology favouring flow separation typically reduces the total drag by reducing skin friction, whereas at fast flows (high Reynolds numbers), flow separation increases the total drag by introducing/enhancing pressure drag. At all flows, flow separation bubbles decrease water renewal at organism surfaces by thickening viscous sublayers, thus reducing exchanges of gases and supply of dissolved nutrients or drifting food particles to the organisms. On the other hand, it decreases the risk of organism surface abrasion by silt transported by water, which can cause considerable damage to the organisms. This interplay of conflicting effects represents an evolutionary dilemma, as simultaneous or sequential adaptation to all these constraints is physically impossible. As a result, the biomechanical and morphological properties of benthic biota often reflect compromises among various physical constraints. The current knowledge on how exactly benthic organisms adapted to physical environments at the water-sediment interface is very fragmentary, and its improvement can only be achieved through combined efforts of ecologists and fluid mechanicists.

From a biological perspective, water-sediment-biota interface regions represent socalled ecotones (i.e., transitional zones) that are characterized by spatial heterogeneities in relation to transported matter, local bed sediment morphologies, flow patterns, and species composition. The heterogeneities in spatial distributions of these features are often the main distinctive attribute differentiating natural environments from constructed ones. To study physical and biological heterogeneities researchers need to overcome a number of conceptual and methodological issues. First, heterogeneities typically occur at multiple scales making it difficult to measure and quantify them due to scale-dependent measurement uncertainties. Second, comparability of the results obtained in laboratory studies and in field conditions may often suffer from high non-linearity of complex transport processes (or unrealistic physical balances) imposing significant scale effects. Third, theoretical and numerical models of heterogeneous environments require identification of appropriate closures for physical quantities emerging as a result of averaging, and to date such closures are very often missing or highly uncertain. 
Organism traits (i.e., any anatomical, morphological, and/or physiological attributes of organisms) play a key role in nearly all processes occurring at biota-water or biotasediment interfaces. Traits are crucial in securing organism capacities to survive in harsh conditions (known as adaptation) often occurring at interfaces, including high levels of spatial heterogeneities, temporal variations, and steep gradients of life-determining factors. Some organisms have the capacity to even modify their environments by employing particular traits (activity known as 'ecosystem engineering'). Organisms-ecosystem-engineers can change physical and biogeochemical processes occurring at interfaces, with possible cascading effects for themselves, other species, and for the whole ecosystem. As a consequence, water sediment-biota interfaces play major roles both in shaping organism traits and evolution and in the functioning of the whole ecosystems.

\section{Interface hydrodynamics}

Hydrodynamic interfaces have been the subject of very active theoretical and experimental studies over the rich history of fluid mechanics and particularly over the last few decades. Such close attention of researchers to interface regions is not surprising, as these regions are ubiquitous features of many flows, often determining the overall mixing, transport, and friction. Indeed, the whole theory of hydrodynamic instabilities has been largely motivated by the intriguing dynamics of interfaces that often exhibit captivating patterns such as water surface waves or ripples on river and coastal sand beds. The range of interfaces studied by modern fluid mechanics is enormous and reflects the expansion of fluid mechanics towards its borders with other disciplines where many interfacial phenomena of different nature from the nano-scale to the astronomical scale occur (e.g., Stone 2010). Ecologically-critical heterogeneous interfaces, which are the subject of this paper, are a typical example emerging at a border of fluid mechanics and aquatic ecology (e.g., Boudreau and Jorgensen 2001). The variety of already-known interfaces in fluid-mechanical applications is so diverse that their comprehensive classification, always useful in research, is not yet available. Nevertheless, we can distinguish several features that may be helpful in interface considerations, i.e., the nature of interfacial phases, interface mobility, and interface dimensionality. Using these three features, hydrodynamic interfaces can be classified as (1) gas-liquid, liquid-liquid, liquidsolid interfaces; (2) fixed or moving interfaces; and (3) two-dimensional or three-dimensional interfaces. Even this oversimplified classification gives twelve types of hydrodynamic interfaces, which in turn can be further subdivided in more subtypes depending on other factors such as scale or prevailing dynamics. To study these interfaces researchers typically start with conventional ideas such as interfacial instabilities, mentioned above, and concepts of boundary layer and mixing layer. 
However, the application of the conventional approaches for interpreting unconventional interfaces may not be straightforward and often presents major challenges. A good example is an interface between a free surface flow and a porous subsurface flow. Clearly, the analysis of such a system can be done at a range of scales from a sub-particle (or void) scale to the whole system scale. Transition from small to large scales requires employment of homogenisation procedures so both 'phases' can be treated at large scales as a continuum. In relation to ecological interfaces this problem has been addressed by researchers dealing with terrestrial canopy aerodynamics, who highlighted the dynamic significance of the inflection point in the mean velocity profile at the canopy top and introduced a mixing layer analogy (and associated Kelvin-Helmholtz instability, KHI), as an expansion of a conventional mixing layer concept (Raupach et al. 1996, Finnigan 2000, Finnigan et al. 2009). For the case of submerged aquatic vegetation, this analogy was first implemented by Nepf's group (e.g., Nepf 2012) and then used in a number of follow-up studies of flowvegetation interactions (e.g., Nezu and Sanjo 2008, Poggi et al. 2004). These studies showed that large-scale mixing layer eddies formed as a result of KHI at the canopy top may play a crucial role in mass and momentum exchanges between a canopy region and flow region above the canopy. Although the mixing layer analogy appears to be directly applicable for aquatic vegetation, there are still a number of issues that require clarification. Some of them suggest that the mixing layer analogy may be a manifestation of a new interfacial mechanism that exhibits unique properties absent in conventional boundary layers and mixing layers. These properties may include (1) the existence of a detached logarithmic boundary layer above a mixing layer at the canopy top (as mixing layer may block access of boundary layer eddies to the canopy layer thus 'detaching' the boundary layer eddies from the bed and destroying the conventional conditions for their formation); (2) significant difference between the convection velocity of large eddies at the canopy top and a local mean velocity, reported for both terrestrial and aquatic canopies (Finnigan 2000, Nepf 2012), although for conventional mixing layers these two velocities should be equal or very close; and (3) monami effect, i.e., wavy motions of a canopy top often observed in natural aquatic canopies, known as 'honami' for terrestrial canopies (de Langre 2008, Nepf 2012). Although motivated by flow-vegetation interface studies, this conjecture may also relate to other flow-biota interfaces such as flow over and within mussels' beds, and to physical interfaces such as flow over and within highly porous sedimentary beds (Nikora 2010).

The proposed conjecture highlights one of the several key challenges that must be addressed: What are the manifestations of the canonical interfacial concepts in aquatic ecosystems? Section 6.1 and Figure 4 illustrates this challenge for the case of aquatic plants, which typically span a wide range of scales from a leaf scale to individual plant to the plant patch mosaic (i.e., an assemblage of plant patches of different shapes and sizes). Another 
challenge is to find What are the combined effects of canonical flow types acting at multiple scales in such flows? This challenge is illustrated with Figure 2 that shows a schematic layering of a gravel-bed flow in which multiple interfaces of different types and at different scales occur. This type of interface, i.e., water-sediment interfaces, is discussed in Section 5.

\section{Ecological role of interfaces}

\subsection{The ecological importance of interfaces: from organism to ecosystem}

Interfaces play a crucial role in ecology, from an individual organism to the whole ecosystem. Interfaces are so important because they represent strong gradients of both resources (e.g., for plants light, $\mathrm{CO}_{2}$, dissolved nutrients, etc.; for animals, $\mathrm{O}_{2}$, food particles, etc.) and waste products and of non-resource abiotic fields (e.g., temperature, density, etc.). Such nonresource fields may strongly affect organisms and whole ecosystems by having an overriding control on fundamental processes (e.g. temperature effect on metabolism). The presence of gradients within ecosystems creates more diverse habitats, both directly and indirectly (via species establishment), thereby supporting the overall bio-diversity. The increased gradients and enhanced biological diversity make interfaces hot spots of ecosystem functioning.

At the ecosystem scale, interfaces are important in determining where organisms can establish and survive. At the water-sediment interfaces in rivers, for example, the sediment provides a relatively stable establishment substrate, whereas the moving water provides a continuous flux of resources and may controls non-resource abiotic fields. A sedentary life form will experience different interactions with its environment than a pelagic life form, which can move (swim). It is also important to consider the changes that occur at different life cycle stages. For example, interfaces may seem less important for pelagic life forms, but at particular times (e.g., the egg stage in fish) there is a requirement for a more sedentary environment with special abiotic conditions (e.g., certain grain size fractions and flow velocities) and access to critical resources (e.g., dissolved oxygen). Thus, hydrodynamic transport through interfaces may control key stages in an organism's life cycle and development.

At the organism scale, organism-environment interfaces are essential to regulating exchange/supply of resources (e.g., $\mathrm{O}_{2}, \mathrm{CO}_{2}$, organic materials, nutrients) and waste products. Depending on organism size and the nature of the surrounding environment, exchange may directly occur via the external surface of the cells (e.g., bacteria, amoeba) or via specialised structures (e.g., leaves, roots, and gills). When resources are limiting, the exchange efficiency can determine species distribution and composition within ecosystems. For example, submerged, floating, and emergent macrophytes differ in their exchange structures, which can 
be specialised for water or air interfaces and this specialism determines their distribution in rivers or lakes.

Whereas most organisms adapt to their physical environment, in some cases they can modify it. Such habitat modification is called ecosystem engineering (Jones et al. 1994), and may alter the number of interfaces, their type and associated gradients (and thereby exchange rates). For example, aquatic vegetation can modify flow turbulence and mean velocity distributions from a leaf scale to the whole vegetation scale, thus altering the exchange rates at plant leaves, inducing deposition of fine sediments within canopies, thereby changing biogeochemical processes. These abiotic alterations can facilitate the establishment of other species (Bruno et al. 2003, Brooker et al. 2008). Ecosystem engineering effects and resultant feedbacks can create both spatial and temporal changes of existing interfaces and even lead to emergence of self-organised landscapes, introducing new interfaces. Hence, the concept of ecosystem engineering needs to be properly considered and accounted for in any studies of interfaces in aquatic ecosystems (Schoelynck et al. 2012).

\subsection{Constraints to life in aquatic ecosystems and the role of interfaces}

Aquatic environments impose three water-specific constraints on organisms, in contrast to terrestrial air environments. First, the high density of water causes the physical (drag) forces acting on organisms to be much stronger than those imposed by wind. Fluctuations of hydrodynamic forces may lead to destabilisation and removal of sessile organisms. The combination of drag and bed erosion around organisms' anchoring systems may further enhance the negative effects (Balke et al. 2013). The relatively high water density means that aquatic organisms have to invest relative few resources in self- supporting structures. Second, compared to non-aquatic environments, oxygen concentration in water is lower, while molecular diffusion of oxygen and other critical substances are slower. The constraints associated with drag and diffusion affect organism shape in opposing senses: increasing an organism's surface area increases the adverse skin friction, but also enhances the valuable substance exchanges. The organisms adapt to these conflicting constraints by employing a variety of strategies. For example, although fish gills have a large surface area for oxygen exchange, the drag is kept to minimum by operculum covering the gills. Flow turbulence imposes control on both skin friction and molecular diffusion by affecting the thicknesses of organism-flow interfaces, namely the viscous sublayer and concentration (diffusive) boundary layers, embedded in the viscous sublayer.

Third, light availability for photosynthesis decreases with water depth. This makes it favorable for autotrophic organisms to grow near the water surface, which is also the place where organisms can have direct access to the air. However, flow velocities and associated 
drag forces tend to be the highest near the water surface. Thus, life in aquatic ecosystems requires adjustments in an organism's morphology and/or physiology, reflecting the biomechanical and physiological constraints (e.g., Bal et al. 2011, Puijalon et al. 2008, 2011). These adjustments are reflected in how organisms adapt to particular hydrodynamic conditions, with interfaces (thin layers adjusting to organism's surface) playing key roles in adaptation strategies.

\subsection{Which interfaces are ecologically important?}

Within aquatic environments, interfaces occur between organism and abiotic medium (i.e., water, air and sediment) and between different components of the abiotic media (i.e., air and water; water and sediment; free-flowing and interstitial water). The importance of the interface between the organism and abiotic medium is obvious, in having a direct impact on an organism's possibility to survive by exchanging substances and ecosystem engineering its environment. The interfaces between different abiotic media are equally important, as they determine the environmental quality in each abiotic compartment. For example, the water-air interface affects the temperature and gaseous content of the water, thereby having an effect on the metabolism of all aquatic organisms. Moreover, it can have a direct effect on specific biological processes such as insect emergence. The free-flowing - interstitial water interface may affect processes like the gas, ion, and particulate-organic-matter exchange, affecting all organisms living in both the water and the sediment.

The exchange rates and the magnitude of gradients across an interface will differ strongly across interface types and this can change spatially and temporally. Interfaces where one phase is moving (e.g., free-flowing water and rigid sessile organism will typically have a greater potential for fast exchange than interfaces where both phases are relatively static (e.g., plant root-sediment). This does not mean, however, that the latter will be unimportant, as i) concentration gradients driving diffusion can be large, ii) sessile organisms may 'slowly move' by replacing old tissues with new ones at a different position and iii) the relatively static interface may offer access to different resources than a mobile interface. Given this complexity, it typically requires case-specific studies to identify which interfaces are most important for the functioning of a specific organism within an ecosystem, and to understand how the organism may alter these interfaces by ecosystem engineering.

\subsection{Identifying gaps in our ecological knowledge of interfaces in aquatic ecosystems}

Whilst studies have shown how organisms meet the demands of living in a spatially heterogeneous and temporally dynamic river environment through a series of compensating adjustments, most compensation analyses focus on single species traits (e.g., Bal et al. 2011, 
Puijalon et al. 2008, 2011). Our challenge is to go beyond the analysis of single traits, and to develop a more holistic understanding of these mechanisms, ideally including the ability of some species to carry out ecosystem engineering. This will inform how organism traits are related to habitat modification and subsequent positive feedbacks to the ecosystemengineering organism (e.g., Bouma et al. 2005).

On a larger scale, there is a need for integrated studies focusing on multiple interfaces and their roles in ecosystem functioning, resilience, and ecosystem services. For example, to answer questions such as (i) does a larger total interface area, or the presence of small hot spot areas, improve the ecosystem functioning and enhance ecosystem services in terms of e.g. water quality or bio-diversity? (ii) To what extent does the overall interface area, or the number of hot spots, determine the ecosystem dynamics and affect an ecosystem's resilience to changes in abiotic drivers such as changes in flow regime or inputs of sediments and associated nutrients? And (iii) can these matters be affected by self-organising processes as recently also identified for aquatic river vegetation (Schoelynck et al. 2012)? Although interfaces are recognized as crucial for the functioning of aquatic organisms and aquatic ecosystems, these key questions cannot be resolved due to lack of insights for up-scaling. As the constraints on life in aquatic environments reflect both physical and ecological processes, the advancement of current knowledge requires integrating the efforts of fluid mechanical and biological researchers.

\section{Water-sediment interfaces}

\subsection{Transport processes in the hyporheic zone}

The mass exchange at the stream-bed interface (i.e., the physical domain connecting the surface flow domain with the groundwater domain) is now much better understood than a couple of decades ago, at least as far as short-term processes are concerned. The permeable sediment layer in the immediate vicinity of the stream boundaries is termed the hyporheic zone (HZ). A comprehensive review of the state of the art in $\mathrm{HZ}$ processes and modelling from and engineering and physical perspective is presented by Boano et al. (2014). This term, however, has quite a general meaning, defining the zone where either specific physical processes (e.g., hydrodynamically induced mass flux) and/or specific ecological properties, representing a distinct ecotone, take place (e.g., Stanford and Ward 1988). The HZ's thickness can, therefore, be defined as ranging from centimetres to hundreds of metres depending on the focus of a particular study.

A key fundamental problem associated with mass transport at the water-sediment interface is the definition of the boundaries of a water body. Transported matter typically 
moves down from a layer of free-flowing water above the bed through complex layers composed of multiple phases such as aquatic plants, periphyton filaments, various microorganisms, and sediments (Figure 3). The fluid content within representative domains (e.g., thin slabs parallel to the bed) decreases from $100 \%$ in the free flow above the bed to $30-$ $40 \%$ in the deep groundwater domain of water-sediment mixture, with regions in between occupied by water-biota and water-biota-sediment phases. The ecologically-critical biota is concentrated at the surface-subsurface interface, and quickly vanishes away from the interface into the sedimentary domain. It is not straightforward to identify a formal stream-bed boundary within this transition zone with multiple interfaces at multiple spatial and temporal scales, particularly from the ecological perspectives. On the other hand, conventional hydraulic engineering defines the stream boundary as the surface of the fluid-sediment interface, effectively reducing the role of plants and other biota to roughness elements. As for mass transport, the zones partially occupied by biota have been traditionally considered as retention or dead zones within the fluid domain, based on their effects as transient mass storage zones. These effects in dispersion models were treated with a variety of empirical or semi-empirical closures. Such an approach, however, is not sufficient in representing ecological systems, as it neglects biologically-significant processes taking place in $\mathrm{HZ}(\mathrm{s})$. The upper hyporheic zones are clearly the most ecologically important regions, as they host roots, micro- to macro- invertebrates, spawned fish eggs, and also play a buffering role in the stream geochemistry (Jones and Mulholland 2000). There is growing evidence that even slow longterm mass exchange processes affecting the subsurface biogeochemistry, including oxygen supply, are of great importance and should not be ignored, as often assumed in transport models of stream mass balance.

Hydrodynamic analysis and experimental observations within HZs have clarified that transport processes acting at time scales of minutes to days are dominated by advective transport resulting from favourable pressure fields induced by local morphological features such as bedforms (a process also referred to as pumping). Finite-volume retention zones, such as vegetated fluid domains and armour sediment layers, produce retention characterized by exponential residence time distributions (RTD), while transport into unbounded domains, like a weakly layered sediment structure, leads to power-law RTDs (Haggerty et al. 2000). While practical engineering applications have favoured the use of transport models using a single retention domain exchanging with the stream (e.g., OTIS Model, Runkel 1998), recent advances have clarified the need to use more complex models, in which the statistics of mass residence time is associated with multiple retention domains through memory functions (CTRW, Boano et al. 2007) or convolution models (STIR, Marion et al. 2008). The next challenge is to incorporate ecological processes into such models (e.g., to predict population dynamics or re-colonisation mechanisms). At the upper part of the scale ladder, the analysis 
of long-term mass transport processes acting at temporal scales of weeks and months and spatial scales of stream reaches and stream-to-catchment domains is also largely undeveloped. There have been few attempts to understand and model transport induced by long-term effects such as floods or other seasonal forcing (e.g., hydrodynamic action of submerged and/or emergent vegetation). A deeper insight is also needed into the biogeochemical role of the hyporheic zone. A comprehensive analysis of the combined effects of inorganic and organic matter and organisms poses a challenge for complex modelling scenarios in the years to come.

\subsection{Grain mobility and interface morphology}

The water-sediment boundary is a key ecological interface as it is the most biologically-active region in aquatic systems (Figure 3, Sections 4.1, 5.1). Although all scales at this boundary may impact on hydraulic and biological processes, direct effects on biota are predominantly linked to smaller, organism-related, scales.

Water-sediment interfaces are often mobile, as individual sediment particles may be entrained and transported during high-flow events. The mobility of sediment can be heavily influenced by organisms, which can either significantly weaken (bioturbation) or strengthen (bioconsolidation) the sedimentary bed. Collectively, entrainment and transport events can modify river bed geometry at a variety of spatial scales, thus affecting the local properties of the interface (e.g., sediment composition, surface roughness, and thus the local flow field) as well as its macro-features, from intra-channel bed forms (e.g., dunes) to the channel planforms (e.g., meanders).

Characteristic time scales of sediment transport processes are linked to these spatial scales, being reflected in variations of the flow intensity (e.g., ratio of the flow velocity to the critical velocity for grain motion) and in the local characteristics of the sediment deposit. Typically, river beds with fine, homogeneous sediments may be mobilised several times a year. The situation is different for gravel-bed rivers, for which most of the sediment matrix is substantially stable, with only finer sediments being mobilised. Thus, during the normal seasonal cycle, gravel-bed rivers could be assumed to be quasi-static with only local sediment transport patterns having the potential to affect the equilibrium. Only extreme long return period hydrological events are typically able to mobilize coarse-sediment fractions at the global (reach) scale, and only these events can change the large-scale bed morphology and severely disrupt the biota. Following such extreme events, bed mobility gradually decreases and the morphology slowly adjusts to the flow, allowing the biota to regain a new state of equilibrium. 
Wide ranges of space and time scales involved in sediment transport processes motivate scale-specific research methodologies and a hierarchy of modelling approaches, from the grain to the river reach scale. However, the understanding of sediment transport in rivers has been hindered by the prevailing intuitive assumption that the flow-sediment boundary can be approximated as a (surface) continuum. In reality, sediment entrainment and transport is a discrete, random process, and the use of the simplifying continuum assumption has severely limited new developments. If understanding of the key physical mechanisms is to be improved, it is essential that focus is placed on the fundamental grain scale and then upscale to larger scales, such as the particle patch/cluster, bedform, and reach scales. This approach should be coupled with a similar consideration for benthic biota that exhibit essentially analogous multi-scale behaviour (from organism to organism and patch to patch mosaics). This research direction should lead to better predictions of channel morphodynamics at scales relevant to engineering and also for biota management.

There is growing recognition that sediment entrainment and the upstream sediment conditions control stability at nearly all scales. Rivers are continuous so even if the local conditions preclude entrainment, the incoming sediment flux can alter the local bed characteristics until a new equilibrium condition is achieved. Several observations have indicated that the incoming flux can have a significant effect on the character of sediment deposits (e.g., Dietrich et al. 1989) and this needs to be appreciated and quantified in consideration of the benthic biota.

Particle mechanics can be broken down into three sequential stages: grain entrainment, motion, and deposition. Buffington and Montgomery (1997) analysed entrainment data collected over eight decades and reported that at high Reynolds number and relative roughness typical for natural rivers, a large range of critical shear stress values were obtained. This could not be explained by the observation method. Field and laboratory experiments indicate that significant stability could be achieved by the formation of cellular type structures on water-worked gravel beds. Thus, the formation of bed structures could significantly modify the flow intensity required to entrain grains of a particular size fraction. Statzner (2012) reported studies in which movement of benthic animals had significantly disrupted the local water-worked bed structure and resulted in a large reduction of the critical bed shear stress, especially for finer sediments. He also reported studies in which some organisms had produced silk to bind sediments together, while others have used their weight to locally consolidate deposits to increase local bed stability. These observations highlight the capacity of some benthic biota to control mobility of water-sediment interfaces, supporting a concept of ecosystem engineering outlined in Section 4. Drake et al. (1988) observed the sporadic nature of fluvial sediment transport and linked this to turbulent sweeps and ejections. Their results inspired many follow-up theoretical and laboratory-based studies of particle 
entrainment that studied the instantaneous local flow field and surface grain arrangement. In terms of statistical descriptions, Grass (1970) first proposed a concept of a joint probability distribution for grain entrainment that accounts for both turbulence and particle arrangement. This concept can incorporate a variety of physical factors, such as particle hiding/exposure and local flow conditions. The joint probability concept can also be applied for flow-biota interactions, i.e., in the stability assessment of sedentary organisms and biologically-worked sediment (bioturbation and bioconsolidation).

After entrainment grain motion is strongly controlled by the momentum gained from the flow. The transport of suspended sediment is thus linked to the turbulence structure and this is reflected in suspended sediment fluxes having a large adaptation length to any flow or bed change. Thus, the local bed condition has little influence on the local suspended sediment flux. Bedload, in contrast, is strongly controlled by the water-sediment interface as the momentum that particles gain during entrainment from the local near-bed flow is strongly influenced by the bed surface structure. Recent studies of grain motion statistics suggests that bed particle motion exhibits diffusive properties, reflecting combined effects of multi-scale bed morphology, turbulence, and mechanisms of particle-particle interactions. This feature of bed particle motion is another example of similarity with benthic biota that also demonstrate diffusive properties in their movements. The diffusive nature of the transport of sediment and biota is ubiquitous to water-biota-sediment interfaces and thus represents a subject for future research.

Finally, while the first two stages of particle transport (i.e., entrainment and motion) have been heavily studied, the third stage, deposition (or disentrainment), has not. Currently the key controlling factors of particle deposition are not clearly understood. They may be associated with the local grain arrangement at the disentrainment site, local turbulence and, for finer particles, with the singularities/wakes associated with larger bed particles, or benthic organisms. Yet, it is the local balance between entrainment and deposition, combined with the sediment fluxes from upstream reaches, that determines the character of the water-sediment interface. If the net streamwise flux is positive, then the surface degrades, whereas if it is negative, the surface aggrades, leaving a historical record of sediment movement in sedimentary deposits. These processes have only been studied semi-empirically and at comparatively large scales. The use of more rigorous grain scale approaches combined with upscaling methods is required to fully examine and properly describe physical, biochemical and biological features and roles of the mobile water-sediment interface. 


\section{Water-biota interfaces}

\subsection{Multi-scale hydrodynamics of water-biota interfaces}

The water-biota interface can be defined at a number of scales, from the scale of the boundary between individual organisms and the water flowing in the vicinity of these organisms to the scales of regions coupling free-flowing water with organisms' communities (e.g., a region at the top of vegetation canopies or mussel patches). In addition to the processes that characterise physical interfaces, the water-biota interfaces also incorporate biological processes that make these interfaces extremely complex. For example, water flow above and around patches of freshwater mussels can comprise a combination of boundary layers, wakes, and mixing layers which are superimposed with jets due to mussel filtering activities. In another example, the posture of an aquatic plant in the flow is a result of complex biomechanical interactions mediated by the balance of drag forces imposed by the flow and the reaction forces of the plant's tissue. The ability of living organisms to engineer their environments makes the water-biota interfaces unique and distinguishes them from the physical interfaces, imposing greater challenges for researchers trying to understand and model them. Living organisms can interact with the flow within a broad spectrum of scales ranging from a sub-organism scale to a scale of a river reach (Nikora 2010, Figure 4).

Organisms tend to form communities, which are often driven by reproduction process. Aquatic plants actively use the mechanism of vegetative propagation that leads to the formation of patches. In communities, individual organisms benefit from the action of the group. At the scale of a patch, the flow can be substantially modified throughout its depth as well as for significant distances downstream. Macro-invertebrates such as bivalve molluscs habitually form patches of considerable size called mussel-beds. The protrusion of mollusc shells alters the flow roughness and results in internal boundary layers that significantly modify transport and mass exchange around the patches. The flow modifications caused by organism patches produce specific large-scale structures such as shear layers, recirculation bubbles, and wakes that can have energy levels several orders of magnitude greater than those at the scale of individual organisms (e.g., in vegetation patches, Nepf 2012).

At the scale of a river reach, patches are organized into mosaics. The tendency to form specific patterns within mosaics reflects the preferences of species to variables such as flow depth and velocity. Patch mosaics also provide a variety of larger-scale flow features, such as stagnation zones, shear layers, and secondary flow structures, and thus promote a diversity of habitats. At the reach scale, these heterogeneities are usually related to morphodynamic features, such as riffle-pool, rapid-pool, or step-pool units. 
Flow-biota interfaces have become the focus of intensive research only during the last few decades. Most of this research has been empirical, and relatively few theoretical approaches are presently known (e.g., Kouwen and Unny 1973, Sand-Jensen 2003, Statzner et al. 2006). The Double-Averaging Methodology (DAM) was proposed as an integrative upscaling framework, which has the potential to address quantitatively the complex issues imposed by a high spatial variability of flow and biota across flow-biota interfaces (Nikora 2010). Over the last decade, there have been several successful examples of the application of DAM to characterize flows over mussel and vegetated beds. Currently, there is a clear research trend to combine the theory of vegetated boundary layers and the mixing layer analogy with biomechanical theories (Niklas et al. 2006, Nikora 2010, Nepf 2012), as described in Section 3. However, progress in the development of these unifying theories is hampered by a lack of knowledge. The most important gaps are the problems of transferring the characteristics of individual organisms to the averaged characteristics at the scale of a patch and a patch mosaic. Phenological cycles and polymorphism of aquatic organisms also contribute greatly to uncertainty in determining the key characteristics to include in combined models. Research needs to focus on these fundamental problems to enable more realistic models of flow-biota interfaces to be developed.

\subsection{Organism traits and interfaces}

Physical, biogeochemical, and biological processes at biota-flow or biota-sediment interfaces are largely mediated by organism traits that are any features measurable at the individual level, from the cell to the whole-organism level. Organism traits can be: (1) anatomical (structures and properties of upper tissues in contact with the environment; (2) morphological (size, architecture); (3) biomechanical (rigidity); (4) physiological (photosynthetic mechanism); (5) phenological (e.g., seasonal growth pattern); and (6) behavioural (mobility, feeding). The combined characteristics of interfaces (enhanced gradients, spatial heterogeneity, temporal variability, intermittency, and multi-scale structure) and living organisms make the trait-mediated processes occurring at the interfaces with organisms different from flow-sediment interfaces, resulting in additional challenges for their study.

First, organism traits relevant to the study of processes at interfaces can be difficult to measure. For instance, some sessile organisms are very flexible (e.g., macrophytes) or highly mobile (e.g., some macroinvertebrates), which makes their shape, motion and position variable and difficult to quantify. Secondly, a given trait, for example the surface area of an organism, has multiple functions that may be antagonistic, as previously described in Section 4. Thirdly, due to the integrated responses of a trait to the multiple stimuli that may affect it, an organism facing a given set of conditions may adopt contrasting strategies, depending on 
other abiotic conditions. For instance, responses to gaseous exchange limitation may vary with water depth, with submerged plants producing terrestrial leaves, where the water is shallow enough, to allow efficient access to gases from air. In some cases, the change of one abiotic factor may even totally impede organism capacity to adapt to another stress (e.g., high nutrient levels reduce the mechanical resistance of plants), inducing an indirect detrimental cascading effect on populations. Fourthly, organism traits and related processes at interfaces also vary spatially and temporally at the biota-flow/sediment interfaces, from a single cell to a whole community spatial scale (as mentioned in Section 6.1) and from very short time scales to evolutionary timescale.

In general, organisms and interfaces can interact in several ways, from unidirectional effects (that is a response from the effect of the organisms on the interface) to bidirectional interactions, and complex feedbacks. Local hydrodynamic and sediment conditions at interfaces may induce organism adaptation which can be a simple use of the local conditions, without modifying them, such as the use of hydraulic shelters for refuge, spawning or feeding. The organisms can also influence the local sediment conditions through increasing or decreasing the likelihood of sediment entrainment. In many cases, organism adaptation also changes the interface. For instance, morphological adaptations can result in locally reduced hydrodynamic forces, thereby minimizing the risk of dislodgement and mechanical damage, or alternatively they may increase flow velocities which maximize feeding and uptake. The modification of conditions at interfaces by organisms (i.e., ecosystem engineering, Jones et al. 1994) may be either passive or active and often depends on organism traits. For example, the passive alteration of flow and intensity of particle trapping by epibenthic organisms depend on organism density, and biomechanical (flexibility) and morphological (size) traits. Active modification of interface conditions also often relies on specific traits, for example, a siphon for pumping animals, and burrowing behaviour or gallery construction for bioturbation.

The study of traits-mediated processes at biota-flow-sediment interfaces presents several important challenges due to the inherent properties of these interfaces. The first challenge is to integrate the relevant questions and approaches carried out at different scales into a general conceptual framework. The possibility of successful upscaling the trait-based approaches requires a precise identification of the contribution of the studies carried out at the different levels and of the gaps between those levels that still need to be filled. As pointed out in the preceding Sections, the study of water-biota interfaces is generally less advanced than the study of water-sediment interfaces. The different types of organisms and their communities (e.g., biofilms, macrophytes, macroinvertebrates) and functional groups (e.g., bioturbating, bioconsolidating animals) have also received different levels of attention due to the specific methodologies required to study them and the theoretical background relevant for each organism type. A second future challenge is therefore to encompass all the interactions 
between the many different types of organisms despite the current gaps in our knowledge of these different groups of organisms.

\section{Wetlands}

The points raised in the preceding sections can be further illustrated with wetlands, which are water bodies exhibiting the widest range of spatial and temporal scales (Figure 1) considered in this paper. More specifically, wetlands are low-laying parts of a catchment characterized by reduced flow velocity and increased water depth and/or width, typically hosting abundant emergent and submerged vegetation. Extensive vegetation domains and flow variability make bio-mechanical properties of wetland plants particularly important. From an environmental protection point of view, wetlands act as natural buffers for contaminants, which are produced by multiple diffuse and point sources (Mitsch and Gosselink 2007), thus providing natural water treatment. Other functions of natural and constructed wetlands include the provision of refuge areas for wild fauna, such as migratory birds, and flood protection through stormwater storage and flow attenuation.

Considering conceptual issues discussed in Sections 2 to 6 , it is apparent that wetlands combine most physical and biological factors relevant to biophysical interfaces, and thus they represent extremely valuable study areas for field experimental research.

Hydrodynamically, wetlands are characterized by complex 3D flow fields combining subdomains of fully developed turbulent flow with extensive subdomains of transitional and quasi-laminar flows, with smooth or sharp transitions between the subdomains, depending on bed geometry and vegetation distribution. As in rivers, both water-sediment and water-biota interfaces introduce specific temporal and spatial scales to momentum and mass transport processes. The key wetland processes vary within temporal scales from seconds to years, and within spatial scales from millimetres to kilometres, both spanning over at least 5 orders of magnitude. The complexity and wide range of interacting processes make wetlands attractive objects for observation and modelling. However, conceptual analysis and modelling of wetlands represent a significant challenge, as currently available knowledge in relation to wetland hydrology, hydrodynamics, and ecology for a range of relevant scales remains scarce. As a consequence, simplified models such as the Continuous Stirred-Tank Reactor (CSTR) dominate practical applications (Kadlec and Wallace 2008). Indeed, constructed wetlands are often hydraulically designed based on the simplest 'well-mixed' assumption, which essentially accounts for the mean residence time (wetland volume/applied discharge) as the dominant design parameter. Among recent approaches, one-dimensional models gained popularity, as they can be applied to main channels by adding empirical exchange terms accounting for the vegetated zones, which are treated as surface storage zones (e.g. Keefe $e t$ 
al. 2004). This type of models allows separation of turbulent flow regions from transitional and quasi-laminar regions, with specific sub-models for these regions as well as for vegetated domains. In relation to models of higher dimensions, the most developed 2D modelling approach available to date adopts shallow water, depth-integrated equations and associated numerical schemes (Musner et al. 2014). However, this 2D approach is only satisfactory when wetlands are not affected by density stratification, i.e., when thermal stratification is negligible. In a summer season the plunging of incoming cold water into a warmer water body can produce density currents and flow short-circuiting, leading to significant reduction of the mean residence time. The ecological implications of such density gradients are likely to be vital, and therefore the adoption of more complex hydrodynamic models, up to threedimensional flow models, represents a goal for future research.

When wetlands are constructed or modified to provide services in addition to their typical functions, an efficiency issue arises and the engineering design and management of the wetlands must to be considered. Examples include the use of constructed wetlands for sediment interception, flood control and/or for tertiary treatment of surface water. In the flood control case, the wetlands must be kept relatively empty in dry conditions to provide attenuation of excess runoff when required. The wetland ecosystem is expected to be in a sort of equilibrium with shallow water depths and slow flow velocities during periods of no rain, but also be able to survive through occasional (up to a few times per year) flooding and excess solute and solid pollutant loads. A sound design and maintenance policy of such wetlands requires further knowledge of the ecosystem's ability to cope with such intermittent hydrologic patterns. In the case of water treatment wetlands, the optimal pollutant removal efficiency strongly depends on the vegetation distribution and plant species composition. The total mass removal is proportional to both flow rate, i.e. hydraulic efficiency, and mass uptake, i.e. treatment efficiency, then a compromise is required regarding what possible strategy should be employed by the control of vegetation traits. The current development of design tools for finding appropriate vegetation distributions involves random field generators and optimization algorithms. The progress, however, is slow due to the lack of factual data on the plant uptake rates for specific pollutants. The improved understanding of the ecological implications of different set-ups and combinations of flows and biota should help with assessment of the viability of restored and constructed wetlands as well as discriminate among different design approaches. These tasks represent an urgent societal issue to be soon addressed by multidisciplinary research teams. In this regard, the ultimate goal for researchers may be to define efficiency metric for wetlands based on measurable physical and ecological quantities, rather than on subjective evaluations. This approach would provide objective indicators on how far wetland efficiency could be improved by anthropogenic measures and help inform the design of the most effective modifications. As it appears to date, modelling of 
wetland complexity may be a goal reachable in a decade, while model calibration through well-organized observation campaigns appears a tougher task to achieve in the near future.

\section{Conclusions}

This forum paper is a collective endeavour of a group of researchers representing different disciplines who are united by a common recognition of the importance of bio-physical interfaces for aquatic systems. To advance this topic, a concerted effort of ecologists, fluid mechanicists, hydrologists, engineers and resource managers is required. This interdisciplinary effort is likely to result in both new exciting discoveries at the discipline borders and a new generation of design and predictive tools which would consider the problem in its entirety rather than reflect discipline-based preferences.

The assessment of the current situation in relation to ecologically-appropriate management of natural and constructed water systems suggests that the progress in this area is delayed due to insufficient knowledge on biophysical interfaces. In this paper, we have attempted to show that nearly all biophysical processes in aquatic systems are driven or controlled by multi-scale interfaces of different origins, including those created by biota themselves. These typically include fluid-fluid, water-sediment, water-biota, and water-biotasediment interfaces. In spite of their importance, however, we still know little about the interfaces' structure, dynamics, and bio-physical roles. Even the key features of the interfaces are not yet properly defined. In Sections 2 to 4 we have shown that one way or another all key conceptual issues of aquatic ecology and eco-hydraulics relate to or depend on biophysical interfaces. In sections 5 to 7 we have tried to outline the current state of affairs in this area, identify knowledge gaps, and highlight the key research challenges for the next decade.

Below we provide a list of interdisciplinary problems which we believe reflect current research needs in this area and which could serve as potential topics for $\mathrm{PhD}$ studies for those who would like to contribute to this field. This list is not comprehensive of course being unavoidably biased by the authors' interests.

1. Qualitative and quantitative assessment of biological and ecological relevance of physical processes occurring at water-sediment interfaces.

2. Development of coherent understanding of coupled ecological, biological, geochemical, and hydrodynamic processes in the hyporeic zones.

3. Development of flow-sediment interface concept that should reflect multiple scales (starting from a grain scale), its mobility, and be based on rigorous definitions of the key processes involved (entrainment, transport, deposition).

4. Identification of the roles of water-sediment interfaces in organism adaptation processes and in general in ecosystem functioning. 
5. Assessment of the applicability of the canonical flow concepts for describing biophysical interfaces and identification of biota-induced flow patterns that are still unknown.

6. Development of appropriate biomechanical models and associated biomechanical parameters for organism species living at water-sediment interfaces.

7. Development of the theoretical frameworks for coupling ecological, biomechanical and hydrodynamic descriptions of the interface biota.

8. Identification of the key organism adaptation mechanisms and development of the biophysical foundations for the ecosystem engineers concept.

9. Development of rigorous up-scaling methodologies for incorporating small-scale biophysical processes occurring at multi-scale interfaces into large scale descriptions relevant to applications (e.g., stream restoration).

10. Development of a general concept of aquatic interfaces accounting for its full bio-geophysical complexity.

\section{Funding}

This work was supported by the Research Executive Agency, through the 7th Framework Programme of the European Union, Support for Training and Career Development of Researchers (Marie Curie - FP7-PEOPLE-2012-ITN), which funded the Initial Training Network (ITN) HYTECH 'Hydrodynamic Transport in Ecologically Critical Heterogeneous Interfaces', N.316546.

\section{Acknowledgements}

The authors acknowledge the support provided by the following partners of the HYTECH project: Christoph Gerstgraser of gerstgraser Ingenieurbürofür Renaturierung; Carlo Someda and Bruno Griffoni of GHT Photonics; Alberto Galli and Matteo Bellinello of SGI Studio Galli; Andrea Rismondo of SELC; and Nicola Berni of Regione Umbria.

\section{References}

Bal K.D., Bouma T.J., Buis K., Struyf E., Schoelynck J., Backx H., Meire P. (2011). Tradeoff between drag reduction and light interception of macrophytes: comparing five aquatic plants with contrasting morphology. Functional Ecology 25, 1197-1205. 
Bodreau, B.P., Jorgensen, B.B. (2001). The Benthic Boundary Layer. Transport processes and biogeochemistry. Oxford University Press, Oxford UK.

Boano F., Packman, A.I., Cortis, A., Revelli, R., Ridolfi, L. (2007). A continuous time random walk approach to the stream transport of solutes. Water Resour. Res. 43:W10425.

Boano, F., Harvey, J.W., Marion, A., Packman, A.I., Revelli, R., Ridolfi, L., Worman, A. (2014). Hyporheic flow and transport processes: Mechanisms, models, and biogeochemical implications. Review of Geophysics, in press.

Bouma T.J., De Vries M.B., Low E., Peralta G., Tánczos I.C., van de Koppel J., Herman P.M.J. (2005). Trade-offs related to ecosystem engineering: A case study on stiffness of emerging macrophytes. Ecology 86, 2187-2199.

Bruno, J.F., Stachowicz J.J., Bertness M.D. (2003). Inclusion of facilitation into ecological theory. Trends in Ecology \& Evolution 18(3), 119-125.

Brooker, R. W., et al. (24 authors) (2008). Facilitation in plant communities: the past, the present, and the future. Journal of Ecology 96(1), 18-34.

Buffington, J.M., Montgomery, D.R. (1997). A systematic analysis of eight decades of incipient motion studies with special reference to gravel-bedded rivers. Water Resour. Res. 33(8) 1993-2029.

de Langre, E. (2008). Effects of wind on plants. Annual Rev. Fluid. Mech. 40, 141-168.

Dietrich, W.E., Kirchner, J.W., Ikeda, H., Iseya F. (1989). Sediment supply and the development of the coarse surface layer in gravel bedded rivers. Nature 340, 215-217.

Drake, T.G., Shreve, R.L., Dietrich, W.E., Whiting, P.J., Leopold L. (1988). Bedload transport of fine gravel observed by motion picture. J. Fluid Mech. 192, 193-217, doi:10.1017/S0022112088001831.

Finnigan, J.J. (2000). Turbulence in plant canopies. Annu. Rev. Fluid. Mech. 32, 519-571.

Finnigan, J.J, Shaw, R., Patton, E. (2009). Turbulence structure above a vegetation canopy. J. Fluid Mech. 637, 387-424.

Global Water Security Declaration. 2013 Chengdu Forum of International Water Organisation. Chengdu, 2013

Grass, A.J. (1970). Initial instability of fine bed sand. J. Hydraul. Div. 96, 619-632.

Haggerty, R., McKenna, S.A., Meigs, L.C. (2000). On the late-time behavior of tracer test breakthrough curves. Water Resour. Res. 36(12), 3467-3479.

Jones, J.B., Mulholland, P.J. (2000). Streams and Ground Waters, San Diego, CA.

Jones, C.G., Lawton, J.H., Shachak, M. (1994). Organisms as ecosystem engineers. Oikos 69: 373-386

Kadlec, R.H., Wallace, S. (2008). Treatment Wetlands. Second Edition. Taylor \& Francis. 
Keefe, S.H., Barber, L.B., Runkel, R.L., Ryan, J.N., McKnight, D.M.,Wass, R.D. (2004) Conservative and reactive solute transport in constructed wetlands. Water Resour. Res. 40, W01201, doi:10.1029/2003WR002130.

Kouwen, N., Unny, T.E. (1973). Flexible Roughness in Open Channels. J. of the Hydraulics Division, ASCE, 99(5), 713-728.

Marion, A., Zaramella, M., Bottacin-Busolin, A.(2008). Solute transport in rivers with multiple storage zones: the STIR model. Water Resour. Res. 44:W10406.

Mitsch, W.J., Gosselink, J.G. (2007). Wetlands John Wiley \& Sons.

Musner, T., Bottacin-Busolin, A., Zaramella, M., Marion, A. (2014). A contaminant transport model for wetlands accounting for distinct residence time bimodality. Journal of Hydrology, $\mathrm{X}(\mathrm{X}), \mathrm{xx}-\mathrm{xx}$

Nezu, I., Sanjou, M. (2008).Turbulence structure and coherent motion in vegetated canopy open-channel flows.J. Hydro-environment Res. 2, 62-90.

Niklas, K.J., Spatz, H-S., Vincent, J. (2006). Plant biomechanics: an overview and prospectus. American Journal of Botany 93, 1369-1378.

Nikora, V. (2010). Hydrodynamics of aquatic systems: an interface between ecology, biomechanics and environmental fluid mechanics. River Research and Applications 26, $367-384$.

Nepf, H.M. (2012). Hydrodynamics of vegetated channels.J. Hydraulic Res. 50, 262-279.

Odling-Smee, F.J., Laland, K.N., Feldman, M.W. (2003). Niche Construction: The Neglected Process in Evolution. Monographs in Population Biology 37. Princeton University Press.

Puijalon S., Bouma T.J., van Groenendael J., Bornette G. (2008). Clonal plasticity of aquatic plant species submitted to mechanical stress: escape versus resistance strategy. Annals of Botany 102, 989-996.

Puijalon S., Bouma T.J., Douady C.J., van Groenendael J., Anten N.P.R., Martel E., Bornette G. (2011). Plant resistance to mechanical stress: evidence of an avoidance-tolerance trade-off. New Phytologist 191, 1141-1149.

Poggi, D., Porporato, A., Ridolfi, L., Alberston, J.D., Katul, G.G. (2004). The effect of vegetation density on canopy sublayer turbulence. Boundary-Layer Meteorol. 111, 565587.

Raupach, M.R., Finnigan, J.J., Brunet, Y. (1996). Coherent eddies and turbulence in vegetation canopies: the mixing layer analogy. Boundary-Layer Meteorol. 78, 351-382.

Runkel, R.L. (1988). One-dimensional transport with inflow and storage (OTIS). A solute transport model for streams and rivers. Water Resour. Invest. Rep. 98-4018, 73 pp., U.S. Geol. Surv., Reston, Va. 
Sand-Jensen, K. (2003). Drag and reconfiguration of freshwater macrophytes. Freshwater Biology 48, 271-283.

Schoelynck, J., T. De Groote, K. Bal, W. Vandenbruwaene, P. Meire, and S. Temmerman (2012), Self-organised patchiness and scale-dependent bio-geomorphic feedbacks in aquatic river vegetation, Ecography 35(8), 760-768.

Stanford, J.A., Ward, J.V. (1988). The hyporheic habitat of river ecosystems. Nature 335, 6466.

Statzner B. (2012). Geomorphological implications of engineering bed sediments by lotic animals. Geomorphology 157-158, 49-65.

Statzner, B., Gore, J.A., Resh V.H. (1988). Hydraulic stream ecology: Observed patterns and potential applications. J. N. Am. Benthol. Soc. 7, 307-360.

Statzner, B., Lamouroux, N., Nikora, V., Sagnes, P. (2006). The debate about drag and reconfiguration of freshwater macrophytes: comparing results obtained by three recently discussed approaches. Freshwater Biology 51, 2173-2183.

Stone, H.A. (2010). Interfaces: in fluid mechanics and across disciplines. J. Fluid. Mech. 645, $1-25$. 


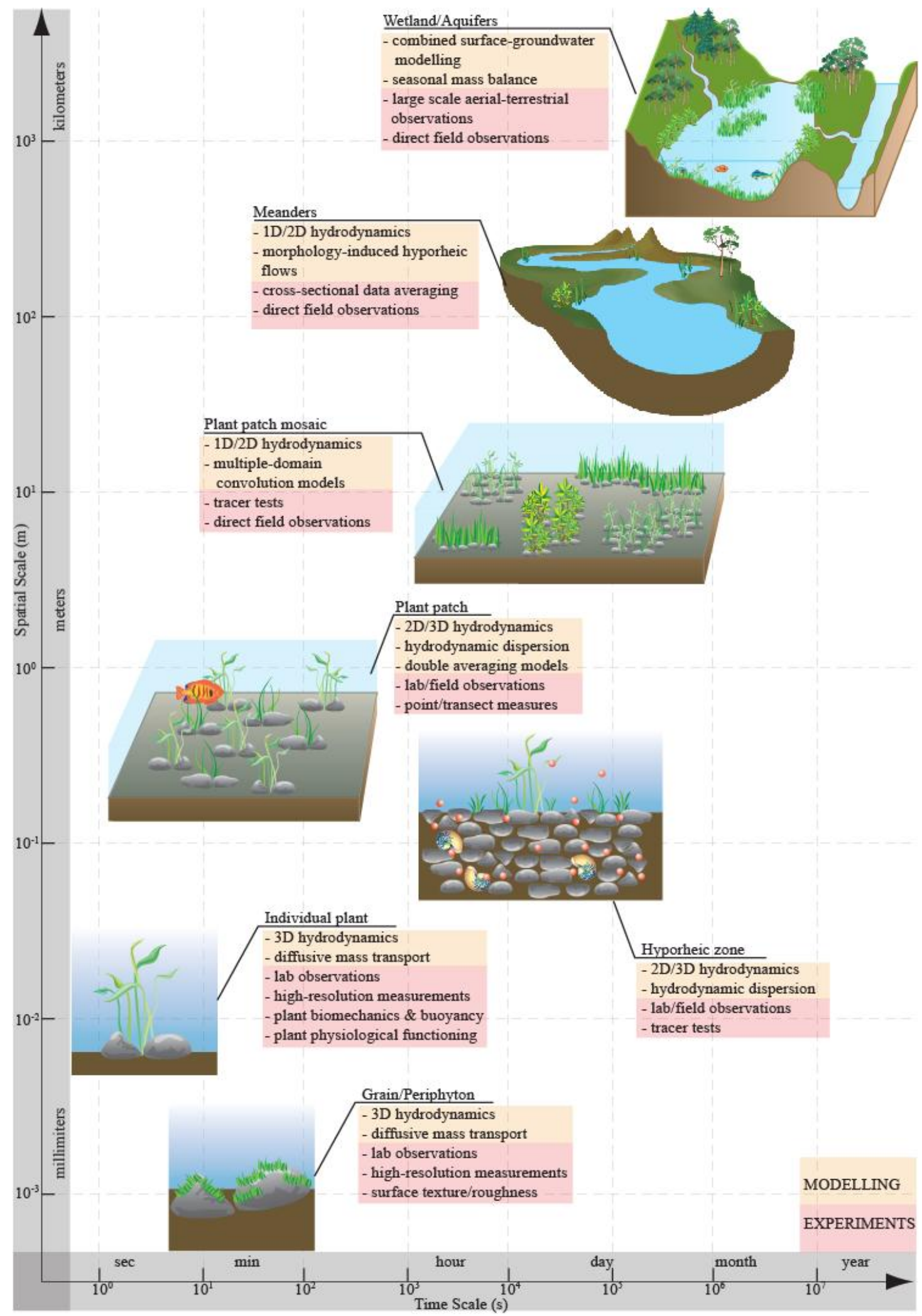

Figure 1 Key domains and fundamental processes in river and wetland eco-hydraulics. The relevant spatial and temporal scales are shown in the vertical and horizontal axes, respectively. The sketch describes the dominant modelling and experimental approach adopted in each domain. 


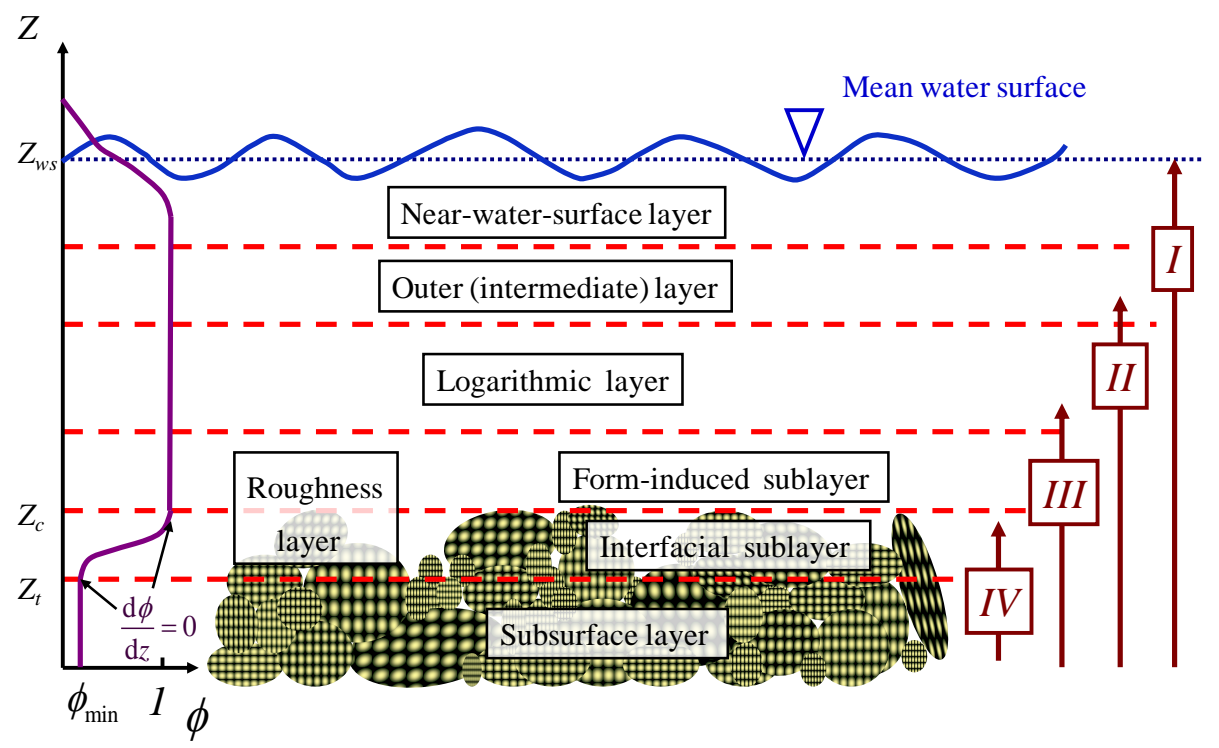

Figure 2 Flow types and flow subdivision into specific regions in gravel-bed flows. The Roman numbers define flows with high (I), intermediate (II), small (III), and partial (IV) submergence. The roughness geometry function $\varphi$ is the ratio of the fluid volume to the total volume of a selected domain (typically a thin slab parallel to the bed, Nikora 2010). 


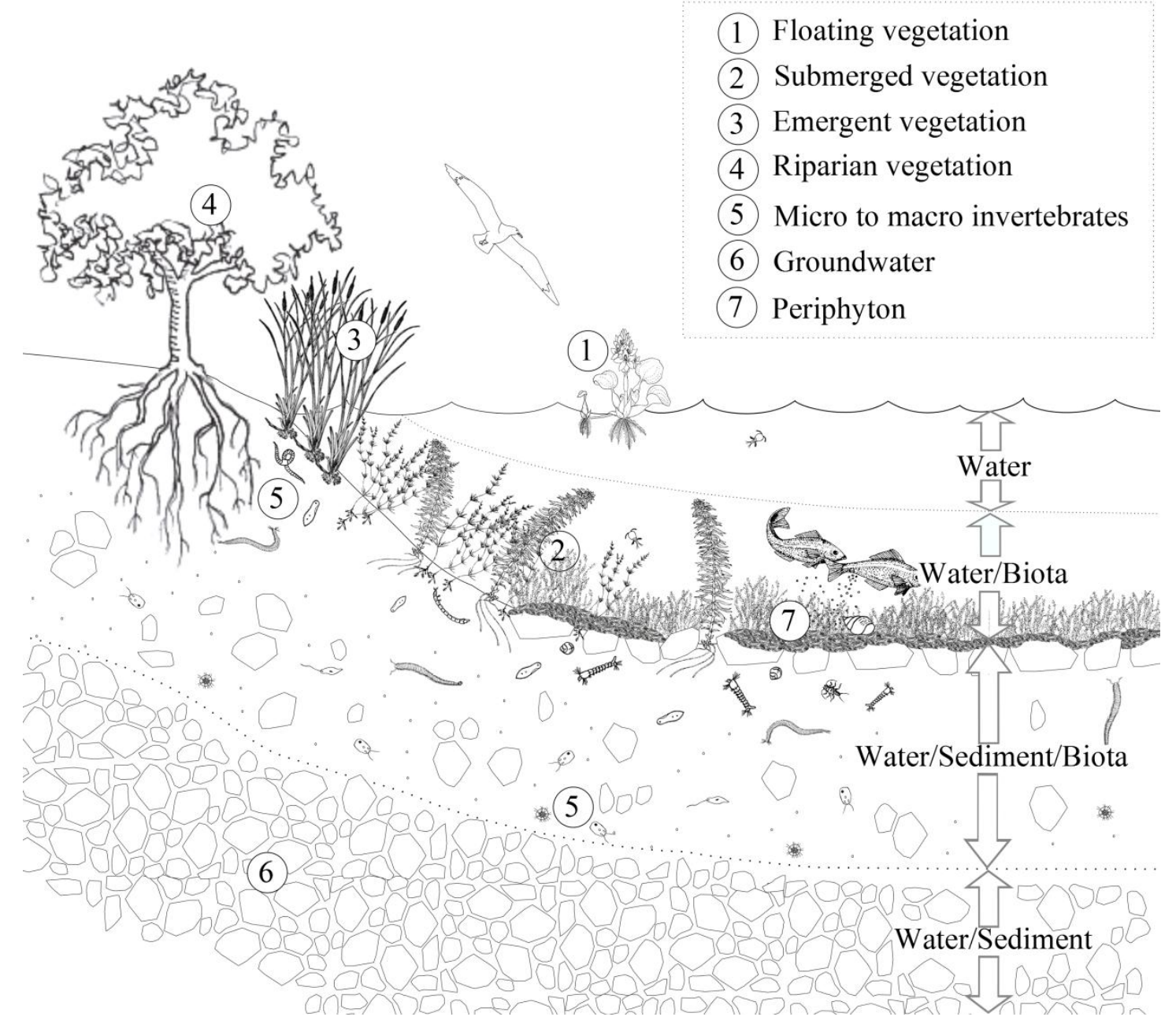

Figure 3 A sketch of the water-biota-sediment interfaces in rivers and wetlands. 
(a)

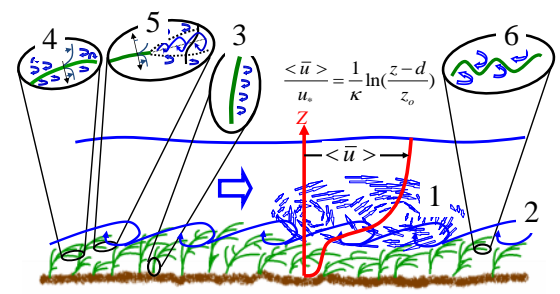

(b)

$\langle\bar{u}\rangle$

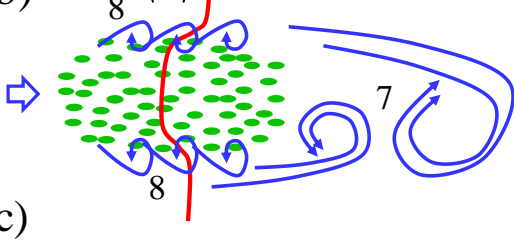

(c)

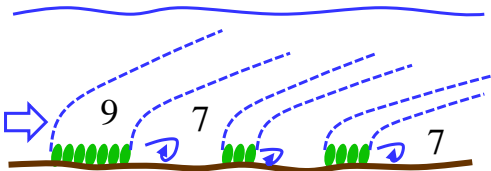

(d)

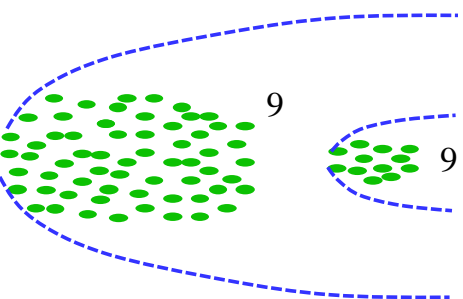

Figure 4 Hypothesised flow patterns in vegetated channels: (a) side view at a patch scale; (b) plan view at a patch scale; (c) side view at a patch mosaic scale; and (d) plan view at a patch mosaic scale. The numbers define: (1) boundary layer depth-scale turbulence; (2) mixing layer canopy-scale turbulence; (3) stem-scale wake turbulence (von Karman vortices); (4) boundary layer leaf-scale turbulence; (5) mixing layer leaf-scale turbulence; (6) leaf-scale wave-generated turbulence; (7) 3D and 2D turbulence associated with wakes and flow separation at a patch scale; (8) 3D and 2D boundary layer and mixing layer turbulence at patch sides aligned with the flow; and (9) 3D and 2D interacting vertical and horizontal internal boundary layers and wakes at the patch mosaic scale (adopted from Nikora 2010) 